\title{
Experimental investigation of cavitation induced air release
}

\author{
Karoline Kowalski ${ }^{1,}$, Stefan Pollak $^{2}$, and Jeanette Hussong ${ }^{3}$ \\ ${ }^{1}$ Ruhr University Bochum, Chair of Process Technology, Universitätsstr. 150, 44801 Bochum, Germany \\ ${ }^{2}$ Ruhr University Bochum, Chair of Particle Technology, Universitätsstr. 150, 44801 Bochum, Germany \\ ${ }^{3}$ Ruhr University Bochum, Chair of Hydraulic Fluid Machinery, Universitätsstr. 150, 44801 Bochum, Germany
}

\begin{abstract}
Variations in cross-sectional areas may lead to pressure drops below a critical value, such that cavitation and air release are provoked in hydraulic systems. Due to a relatively slow dissolution of gas bubbles, the performance of hydraulic systems will be affected on long time scales by the gas phase. Therefore predictions of air production rates are desirable to describe the system characteristics. Existing investigations on generic geometries such as micro-orifice flows show an outgassing process due to hydrodynamic cavitation which takes place on time scales far shorter than diffusion processes. The aim of the present investigation is to find a correlation between global, hydrodynamic flow characteristics and cavitation induced undissolved gas fractions generated behind generic flow constrictions such as an orifice or venturi tube. Experimental investigations are realised in a cavitation channel that enables an independent adjustment of the pressure level upstream and downstream of the orifice. Released air fractions are determined by means of shadowgraphy imaging. First results indicate that an increased cavitation activity leads to a rapid increase in undissolved gas volume only in the choking regime. The frequency distribution of generated gas bubble size seems to depend only indirectly on the cavitation intensity driven by an increase of downstream coalescence events due to a more densely populated bubbly flow.
\end{abstract}

\section{Introduction}

Hydrodynamic cavitation in liquid flows can be produced by a pressure drop behind a flow constriction. Since most liquids used in technical applications contain dissolved gases both vaporous cavitation and degassing processes may take place simultaneously at different intensity depending on the pressure conditions and solubility of gases in liquids. The cavitation process is influenced by e.g. the liquid properties and often takes place as heterogeneous cavitation at nuclei such as impurities or the liquid-solid interface [1].

The two phase flow has an effect on the density, speed of sound and compressibility of the flowing liquid. This can lead to noise, vibrations and fluctuation of the mass flow rate in hydraulic systems and lower the efficiency of the system. Simulations of air release are mostly based on the assumption of a diffusion process which fails to make quantitative predictions on cavitation induced degassing [2]. Most flow cavitation experiments are realised in small scale and/or rectangular cross sectional channels, e.g. [3-6]. Optical measurements of air release provoked by cavitation in a rectangular microchannel of testing oil were performed by Freudigmann et al. [3]. As commonly known, cavitation phenomena cannot be simply scaled up [7]. A rectangular channel cross section enables a better optical access but leads to topology related corner flow and cavitation behaviour. Due to this fact, the experiments described in this paper are restricted to rotationally symmetric cavitation flows in generic geometries.

The general hydrodynamic behaviour of a noncavitating orifice flow is well described by the Bernoulli equation. When choked flow conditions are reached no further increase in volume flow rate can be obtained and the mass flow rate stays at a constant value. The onset of a choked flow condition depends on the choice of upstream parameters only [8].

The dimensionless pressure drop can be described through the cavitation number $\sigma$ which is given by Nurick and others [9].

$$
\sigma=\frac{P_{\text {in }}-P_{v}}{P_{\text {in }}-P_{\text {out }}}
$$

$P_{\text {in }}$ denotes the inlet pressure and $P_{v}$ the vapour pressure. The pressure difference $P_{\text {in }}-P_{\text {out }}$ is measured over the flow constriction. Decreasing cavitation numbers correspond to an increase in cavitation probability or intensity, respectively.

\section{Experimental set-up}

This section is divided into two parts. In section 2.1 the experimental set-up and its hardware components are described. In the following sections 2.2 and 2.3 details are given on the measurement procedure for flow characteristics and the optical measurements. 


\subsection{Cavitation channel}

In the experimental set-up (Fig. 1) two stainless steel pressure vessels (585 1 volume, 0 to 10 bar pressure range) are integrated in a closed fluid cycle. In the first vessel $V_{1}$ compressed air is used to set the pressure level $\mathrm{P}_{1}$. The liquid can be circulated through a bypass BP to reach a faster gas saturation of the liquid using a centrifugal pump (MXH 803, Linn Pumpen). The second vessel $V_{2}$ can be either evacuated down to 0.2 bar using a vacuum pump (VACFOX VGD 10, Ehrler \& Beck $\mathrm{GmbH}$ ) or it can be set to higher pressures using compressed air. Different constrictions, e.g. orifices or venturi tubes can be placed in the test section. Due to the pressure difference between both vessels the overall pressure level $P_{\text {in }}$ and volume flow rate $\dot{V}$ can be set independently in the measurement section.

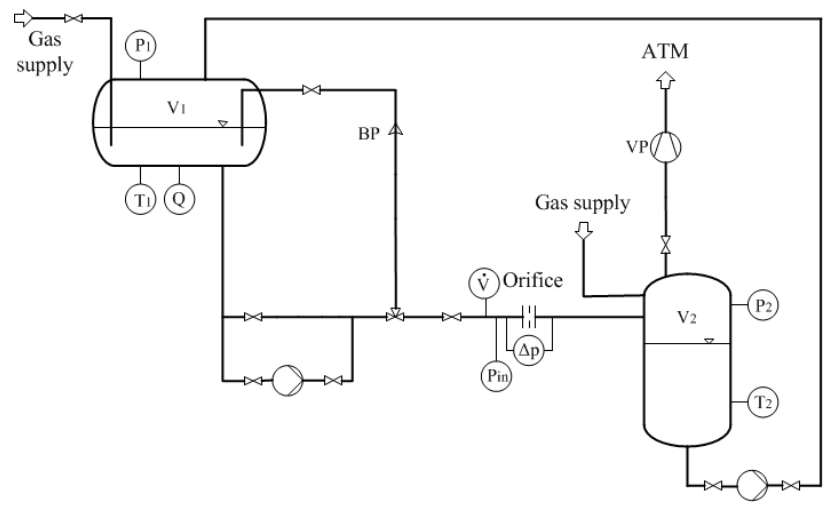

Fig. 1: Draft of the experimental set-up with pressure, flowrate, temperature and gas saturation sensors

The experiments are executed as a batch process to avoid influences of fluctuating gas content upstream of the test section. For every operation point the vessel pressures $P_{1}$ and $\mathrm{P}_{2}$, as well as corresponding vessel temperatures $\mathrm{T}_{1}$ and $\mathrm{T}_{2}$, the inlet pressure $P_{i n}$, the oxygen content $\mathrm{Q}$ in the first vessel, the volume flow rate $\dot{V}$ and the pressure difference $\Delta \mathrm{p}$ at the orifice are recorded with $100 \mathrm{~Hz}$ for 2 minutes.

For examination of the orifice flow characteristics, measurements are performed for different inlet pressure levels $P_{i n}$ at which the outlet pressure $\mathrm{P}_{2}$ is lowered stepwise.

The orifice assumes a bore diameter of $3 \mathrm{~mm}$ with a $45^{\circ}$ chamfer of approximately $0.63 \mathrm{~mm}$ at the inlet and a conical outlet of $60^{\circ}$ (see Fig. 2). The flow direction is from left to right.

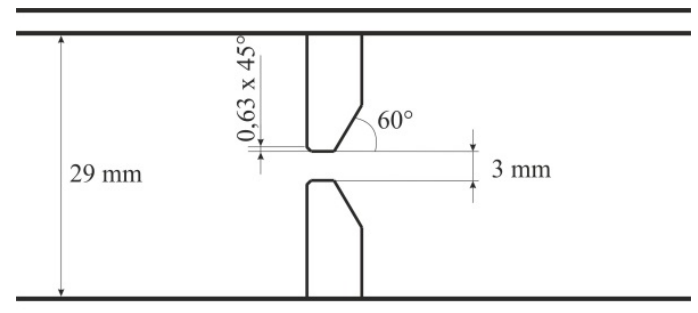

Fig. $\overline{2 \text { : Sketch of the orifice geometry used in the experiments }}$

\subsection{Flow characteristics}

For each flow characteristic the inlet pressure is set to a fixed value. The measurement sequence is started with a maximum outlet pressure, which is decreased in 1 bar steps or less after every measurement point down to 0.2 bar. For every operation point data is recorded after the flow is fully developed and stationary flow conditions are reached. In the experiments the cavitation number is lowered in every measurement sequence by reducing the outlet pressure $\mathrm{P}_{2}$.

\subsection{Optical measurements}

Flow characteristics and optical measurements were performed simultaneously. Shadowgraphy images were taken behind the orifice in an optically accessible test section using a CCD camera (ImagerProSX, LaVision) and a pulsed LED light source (Backlight blue, LaVision) as indicated in Fig. 3a.

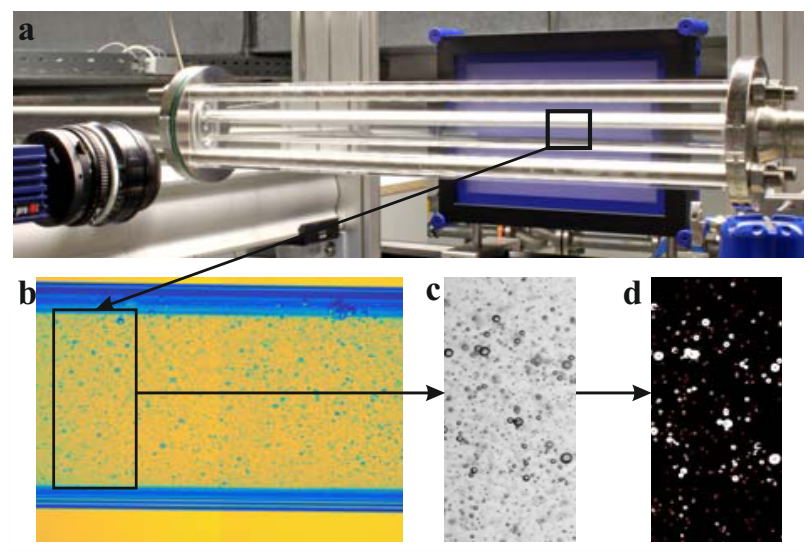

Fig. 3: a) Test section with LED light source and CCD camera, b) representative FOV of $40.64 \times 34.03 \mathrm{~mm}^{2}$ located $30 \mathrm{~mm}$ downstream of the orifice, c) region of interest selected for statistical evaluation, d) postprocessed image, detected bubble centers are marked red

Images are taken with a $50 \mathrm{~mm}$ focal distance lens (Nikon, Nikkor Lens AF $50 \mathrm{~mm} \mathrm{f} / 1.4 \mathrm{D}$ ) and a 4x closeup lens. For every operation point, 200 images are recorded with a spatial resolution of $0.0166 \mathrm{~mm}$ per pixel covering a field of view (FOV) of $40.64 \times 34.03 \mathrm{~mm}^{2}$. Images are taken $30 \mathrm{~cm}$ behind the orifice exit in a cavitation free zone to record air bubbles only. Due to total reflection a section of approximately $4 \mathrm{~mm}$ of the upper and lower part cannot be accessed optically (dark areas in Fig. 3b).

The images are postprocessed by a self-written MATLAB algorithm. The bubble size distribution and bubble density for every operation point were determined. For preprocessing a sliding average filter of $2 \times 2$ pixel filter length was used to reduce the salt and pepper noise in the images. Hence, a constant image threshold was applied for a black-white transformation. Afterwards, all objects in the image plane were filled and labelled. Only objects with more than 4 pixels area size are used for a statistical analysis. For every measurement point shown in Fig. 4 the mean bubble size distribution 
was extracted from 200 images, corresponding to $40 \mathrm{~s}$ recording sequence. Furthermore, the ratio $\alpha$ between the area occupied by bubbles $A_{\text {bubbles }}$ and the total image area $A_{\text {image }}$ is depicted as measure for the released air volume fraction at different operation points.

$$
\alpha=\frac{A_{\text {bubbles }}}{A_{\text {image }}}
$$

\section{Results}

In this section flow characteristics and degassed volume fractions are measured for a cavitating orifice flow.

\subsection{Flow characteristics}

In Fig. 4 the hydraulic characteristics of the set-up with an orifice of $3 \mathrm{~mm}$ bore diameter are shown. Three different inlet pressure levels of 5.8, 7.8 and 9.8 bar are depicted. Each measurement was repeated three times.

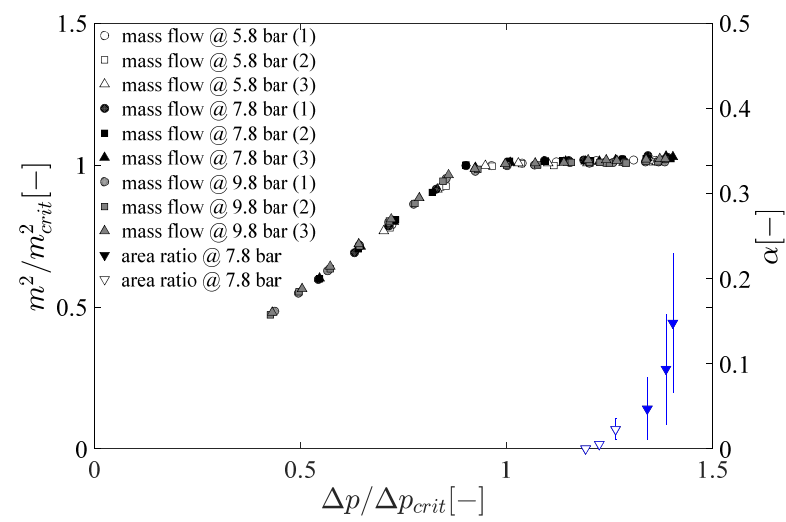

Fig. 4: Flow characteristics for inlet pressures $P_{\text {in }}=9.8$ bar, 7.8 bar, 5.8 bar (black labels) and results of air release fraction $\alpha$ for an inlet pressure of $\mathrm{P}_{\text {in }}=7.8$ bar (blue labels)

Normalised squared volume flow rates $\dot{m}^{2} / \dot{m}_{\text {crit }}^{2}$ are plotted as a function of the normalised pressure differences $\Delta \mathrm{p} / \Delta \mathrm{p}_{\text {crit }}$ across the orifice. Critical values refer to the shift from the so called Bernoulli regime to the choked flow regime, apparent through a kink in the flow characteristics. Within the Bernoulli regime, the squared mass flow rate scales with the pressure drop across the flow constriction. The critical mass flow rate at which choking takes place depends on the inlet pressure. A standard deviation of mean mass flow rates $s(q)$ of less than $0.23 \%$ was determined based on the arithmetic mean $q$ and standard deviation $s\left(q_{k}\right)$ of $n$ individual observations $q_{k}$ (see [10]).

$$
\begin{gathered}
q=\frac{1}{n} \cdot \sum q_{k} \\
s\left(q_{k}\right)=\sqrt{\frac{1}{n-1} \sum\left(q_{k}-q\right)^{2}} \\
s(q)=\frac{s\left(q_{k}\right)}{\sqrt{n}}
\end{gathered}
$$

The critical values for the mass flow rate $\dot{m}_{\text {crit }}^{2}$ and the pressure difference $\Delta \mathrm{p}_{\text {crit }}$, respectively are given in Tab. 1.

Table 1: Critical values for choked flow conditions

\begin{tabular}{l|l|l|l|l}
$\begin{array}{l}\mathrm{P}_{\text {in }} \\
{[\mathrm{bar}]}\end{array}$ & $\begin{array}{l}\Delta \mathrm{p}_{\text {crit }} \\
{[\mathrm{bar}]}\end{array}$ & $\begin{array}{l}\dot{m}_{\text {crit }}^{2} \\
{\left[(\mathrm{~g} / \mathrm{s})^{2}\right]}\end{array}$ & $\begin{array}{l}\mathrm{P}_{2 \text { crit }} \\
{[\mathrm{bar}]}\end{array}$ & $\begin{array}{l}\sigma_{\text {crit }} \\
{[-]}\end{array}$ \\
\hline \hline 5.8 & 4.0 & 34000 & 2 & 1.44 \\
\hline 7.8 & 5.4 & 45500 & 2.4 & 1.43 \\
\hline 9.8 & 6.9 & 57750 & 3 & 1.42
\end{tabular}

For increasing inlet pressures $P_{\text {in }}$ the critical pressure drop $\Delta \mathrm{p}_{\text {crit }}$, the critical squared mass flow rate $\dot{m}^{2}$ crit and critical outlet pressure $\mathrm{P}_{2 \text { crit }}$ increase. The critical cavitation number $\sigma_{\text {crit }}$ shows a slight decrease but is nearly constant for the three examined inlet pressures. This is expected as only a small variation of inlet pressures $P_{\text {in }}$ was examined. The cavitation number is calculated as described in Eq. 1 with $\Delta \mathrm{p}$ as the pressure difference at the flow constriction.

The experiments were carried out for cavitation numbers between $1.03<\sigma<3.34$. Due to a critical cavitation number of the system of approximately 1.4 , non-cavitating flows for $\sigma>\sigma_{\text {crit }}$ and cavitating flows for $\sigma<\sigma_{\text {crit }}$ were realised.

\subsection{Optical measurements}

In this section, results obtained from optical measurements are presented. Representative shadowgraphy images for an inlet pressure $P_{\text {in }}=5.8 \mathrm{bar}$ and three different outlet pressures $P_{\text {out }}=1.4,0.7$ and 0.2 bar are shown in Fig. 5a-c. The region of interest covers 500 pixels in width and 1250 pixels in height corresponding to $8.3 \times 20.75 \mathrm{~mm}^{2}$.
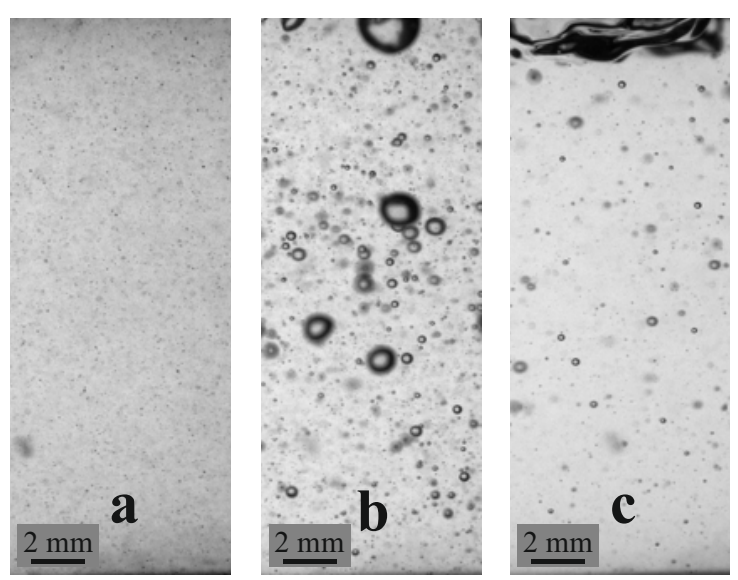

Fig. 5: Representative shadowgraphy images of a liquid flow $30 \mathrm{~mm}$ downstream of the orifice with an inlet pressure of $\mathrm{P}_{\text {in }}=5.8 \mathrm{bar}$ and oxygen content of $\mathrm{Q}=9.5 \mathrm{mg} / 1 \pm 0.3 \mathrm{mg} / \mathrm{l}$ : a) $\mathrm{P}_{2}=1.4$ bar, b) $\mathrm{P}_{2}=0.7$ bar, c) $\mathrm{P}_{2}=0.2$ bar

A comparison of figures $5 \mathrm{a}$ and $\mathrm{b}$ show that the air bubble size increases with rising cavitation intensity. Due to buoyancy forces, bigger bubbles rise faster to the top of the pipe. Therefore the mean bubble size and density in the bulk tends to decrease downstream of the cavitation region, while larger air volumes accumulate at 
the upper wall as shown in Fig. 5c. The area ratio $\alpha$ of released air within the measurement volume is plotted for $P_{i n}=7.8$ bar in Fig. 4. Optical measurements show an increase of bubble filled areas with increasing pressure difference. The air release in the observed area begins later than the choked flow conditions are reached. The standard deviation of the area ratio $\alpha$ seems to grow with increasing pressure drop due to inhomogeneous bubble coalescence. Operation points at which such distinct air accumulation was observed are labelled with filled markers as systematic errors of $\alpha$ are expected due to a limited optical access within the chosen FOV. A first estimate of the buoyancy related error of $\alpha$ was performed. For this, we assume that bubbles move with the centreline velocity $u_{c}$ of a fully developed turbulent flow utilizing a drag coefficient of $c_{D}=2 F_{d} / \pi \rho u^{2} R^{2}[11]$. Based on a force balance of buoyancy $\left(F_{b}\right)$ and drag force $\left(F_{d}\right)$ a critical bubble radius was determined to:

$$
R=\sqrt{\frac{3 \cdot \mu \cdot u_{c}}{\Delta \rho \cdot g}}
$$

Here, $\Delta \rho$ denotes the density difference, $g$ the gravity constant, $\mu$ the water viscosity and $u_{c}$ the centreline velocity. The Reynolds number is calculated as $R e=(\rho \cdot d \cdot u) / \mu$. Based on the centreline velocity of a fully developed turbulent flow $\left(u_{c} \approx 1.2 \cdot u_{b u l k}=0.3 \mathrm{~m} / \mathrm{s}\right)$ bubbles of $R \geq 0.3 \mathrm{~mm}$ are expected to rise to the top of the pipe before reaching the downstream measurement section. As in the depicted FOV bubbles with a radius greater than the critical radius are detected in the bulk flow we assume that a delayed formation of these bubbles or a delayed coalescence further downstream the orifice takes place.

The image analysis is applied for operation points in the choked flow regime. As no released air bubbles could be observed close to the critical mass flow rate (see Fig. 4), we anticipate that this is either due to a limited spatial resolution or a diffusion process taking place such that small bubbles get into solution before entering the depicted FOV. Based on the spatial resolution limit a bubble has to exceed a minimum radius of $R_{\text {min }}=0.0132 \mathrm{~mm}$, corresponding to a bubble image area of 4 pixel, to enter the statistical analysis. Based on the bulk velocity and time scales a conservative estimate can be made to determine the minimal radius of bubbles created behind the orifice that decrease their size during convection due to diffusion driven solution. For this, we assume that one bubble with radius $R_{0}$ at the orifice exit is filled with air only. This bubble is surrounded by degassed water (gas concentration $\left.c_{0}=0 \mathrm{mg} / \mathrm{l}\right)$. The time for the bubble to arrive in the measurement section amounts to approximately $t=1 \mathrm{~s}$. The minimal bubble radius has to be $R_{\text {min }}$, for the given spatial resolution, to be analysed in the postprocessing. Using equation 5 the radius $R_{0}$ of the bubble can be calculated [12].

$$
R_{\min }=R_{0}^{2}-\frac{2 \cdot \kappa}{\rho} \cdot\left(c_{s}-c_{0}\right) \cdot t
$$

The diffusion coefficient is assumed to be $\kappa=2.1 \cdot 10^{9} \mathrm{~m}^{2} / \mathrm{s}$, the density $\rho=1.189 \mathrm{~kg} / \mathrm{m}^{3}$ and the saturation concentration of oxygen in water $c_{S}=9 \mathrm{mg} / \mathrm{l}$. Thus, a bubble with an initial radius of $R_{0} \leq 15.8 \mu \mathrm{m}$ would dissolve to an extent that it is neglected in the evaluation procedure.

Fig. 6 displays the influence of the cavitation number on the volume faction of released air. Results are shown for an inlet pressure $P_{i n}=7.8$ bar whereas results for the buoyancy dominated regime are labelled with filled markers. The amount of released gas grows with decreasing cavitation number $\sigma$ as the latter is associated with the increase in cavitation intensity. The present results show a similar trend to studies by Iben et al. [13], who measured an exponential increase in released gas inside a high pressure microfluidic device. However, for the present set-up studies at different downstream positions and under stereoscopic viewing angles are required in the future to gain statistically more reliable results for cavitation numbers below $\sigma=1.1$ allowing to elaborate on the scaling behaviour in more detail.

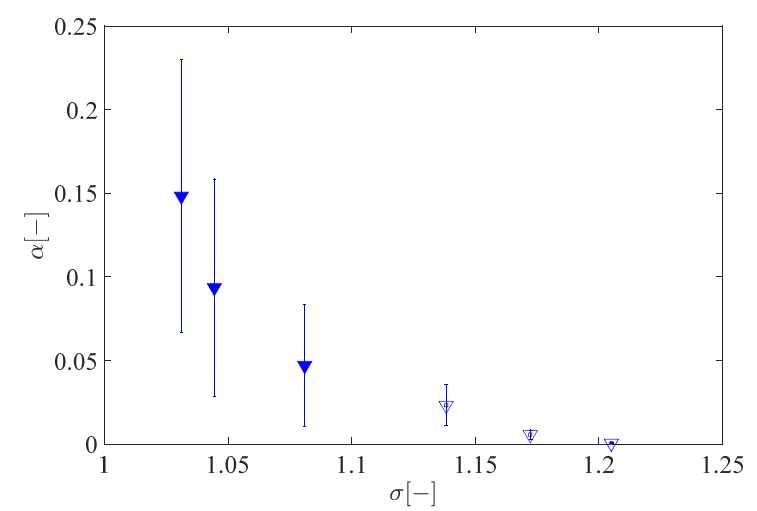

Fig. 6: Results of air release fraction as function of cavitation number for an inlet pressure of $\mathrm{P}_{\mathrm{in}}=7.8 \mathrm{bar}$

Released air fraction values were calculated from bubble frequency and size distributions. Corresponding bubble frequency histograms are plotted in Fig. 7 as function of bubble radius $\mathrm{R}_{\mathrm{b}}$ for different outlet pressures.

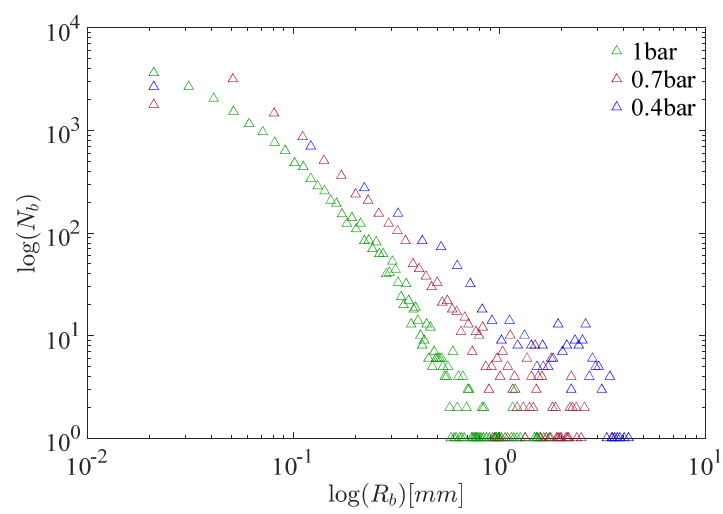

Fig. 7: Number of gas bubbles $\left(\mathrm{N}_{\mathrm{b}}\right)$ as function of bubble radius $\left(\mathrm{R}_{\mathrm{b}}\right)$ for 200 recordings, $\mathrm{P}_{\mathrm{in}}=7.8 \mathrm{bar}$ and outlet pressures $\mathrm{P}_{2}=1,0.7$ and 0.4 bar

The bubble radius ranges from 0.02 to $4.23 \mathrm{~mm}$. The frequency spectrum in the range between $0.03 \mathrm{~mm}<\mathrm{R}_{\mathrm{b}}<0.8 \mathrm{~mm}$ is very similar for all outlet pressures. Differences can be seen for the smallest radius 
$\mathrm{R}_{\mathrm{b}}=0.02 \mathrm{~mm}$, where the frequency of bubbles for an outlet pressure $P_{\text {in }}=1 \mathrm{bar}$ (which corresponds to $\sigma=1.14)$ is $27 \%$ higher than for lower outlet pressures $(\sigma=1.04,1.03)$. Main differences can be observed for larger bubble radii which have a strong influence on the results of the area ratio $\alpha$. For decreasing outlet pressures, and consequently also decreasing cavitation number, the frequency of bubbles with radii $R_{b}>0.8 \mathrm{~mm}$ increases. A general trend from smaller to higher bubble sizes with increasing cavitation intensity can be observed.

\section{Conclusions}

In the present study, a correlation between hydrodynamic behaviour and released air fraction of a cavitating orifice flow in the choking regime is investigated. For incipient choked flow conditions no air release could be observed up to approximately 1.2 times the critical pressure drop. A further decrease of the outlet pressure is associated with increasing air bubble volume fraction and a change in bubble size frequency. In alignment with results from Iben et al. [13] an increase in released air bubble fraction was observed for decreasing cavitation numbers.

In future, an extended, thorough experimental study will allow to gain statistical results of gas volume fractions also for lower cavitation numbers. For this, results have to be obtained in different measurement plains and viewing angles to cover the full cross sectional area of the flow. Detailed studies will provide further information on the dissolving process and representative time scales of the coalescence dynamics. In an attempt to find universal scaling relations of cavitation driven air release behind different generic flow constrictions studies will be repeated for different flow constriction geometries such as venturi tubes.

\section{Acknowledgment}

This research is supported by the "Competence Center of Hydraulic Fluid Machinery”.

\section{References}

1. F. R. Young, Cavitation, World Scienific, (1989)

2. N. I. Kolev, Multiphase Flow Dynamics 4, Springer (2011)

3. H. A. Freudigmann, U. Iben, P. F. Pelz, Journal of Physics 656, 012113 (2015)

4. P. F. Dunn, F. O. Thomas, M. P. Davis, I. E. Dorofeeva, Physics of Fluids 22, 117102 (2010)

5. C. Mishra, Y. Peles, Journal of Microelectrochemical Systems 14 (5), 987 - 999 (2005)

6. K. Ramamurthi, K. Nandakumar, Flow Measurement and Instrumentation 10, 133-143 (1999)

7. C. E. Brennen, Multiphase Fundamentals of Multiphase Flows, Cambridge University Press, (2005)

8. I. H. Shames, Mechanics of fluids, 3 ed., (1992)

9. W. H. Nurick, Journal of Fluids Engineering 98 (4), 681-687 (1976)

10. Guide to the expression of uncertainty in measurement, JCGM 100, (2008)

11. R. Clift, Bubbles, Drops, and Particles, Academic Press, (1978)

12. L. Liebermann, Journal of Applied Physics 28, 205 (1957)

13. U. Iben, F. Wolf, H.-A. Freudigmann, J. Fröhlich, W. Heller, Exp. Fluids 56, 114 (2015) 